International Journal of Pure and Applied Mathematics

Volume 111 No. 3 2016, 361-372

ISSN: 1311-8080 (printed version); ISSN: 1314-3395 (on-line version)

url: http://www.ijpam.eu

doi: 10.12732/ijpam.v111i3.1

\title{
ON THE CONNECTIONS BETWEEN THE PERMANENTS OF SOME TRIDIAGONAL MATRICES AND THE PELL AND JACOBSTHAL NUMBERS
}

\author{
Ahmet Öteleş \\ ${ }^{1}$ Department of Mathematics \\ Faculty of Education \\ Dicle University \\ TR-21280, Diyarbakir, TURKEY
}

\begin{abstract}
In this paper, we consider two $n \times n$ tridiagonal matrix families. Then we study the relationships between permanents of these families and Pell and Jacobsthal sequences. Moreover, we present two Maple 13 procedures that give connections between these permanents and integer sequences.
\end{abstract}

AMS Subject Classification: $11 \mathrm{~B} 83,15 \mathrm{~B} 36,11 \mathrm{C} 20$

Key Words: Pell sequence, Jacobsthal sequence, tridiagonal matrix, permanent

\section{Introduction}

The well-known Pell sequence $\left\{P_{n}\right\}_{n=0}^{\infty}$ and Jacobsthal sequence $\left\{J_{n}\right\}_{n=0}^{\infty}$ are defined by the recurrence relation

$$
\begin{aligned}
P_{n} & =2 P_{n-1}+P_{n-2}, & & P_{1}=1, \text { and } P_{2}=2, \\
J_{n} & =J_{n-1}+2 J_{n-2}, & & J_{1}=J_{2}=1,
\end{aligned}
$$

for $n \geq 2$. These sequences are respectively named $A 000129$ and $A 001045$ in

Received: $\quad$ February 20, 2016

Revised: $\quad$ November 11, 2016

Published: $\quad$ December 16, 2016 (c) 2016 Academic Publications, Ltd. url: www.acadpubl.eu 
$[1]$.

Binet formulas of these sequences are

$$
P_{n}=\frac{(1+\sqrt{2})^{n}-(1-\sqrt{2})^{n}}{2 \sqrt{2}}
$$

and

$$
J_{n}=\frac{2^{n}-(-1)^{n}}{3}
$$

The permanent of an $n$-square matrix $A=\left[a_{i j}\right]$ is defined by

$$
\operatorname{per} A=\sum_{\sigma \in S_{n}} \prod_{i=1}^{n} a_{i \sigma(i)},
$$

where the summation extends over all permutations $\sigma$ of the symmetric group $S_{n}$. The basic properties of the permanent can be found in [2]. The permanent of a matrix is analogous to the determinant, where all of the signs used in the Laplace expansion of minors are positive (see [3]). Permanents have many applications in physics, chemistry and electrical engineering.

Let $A=\left[a_{i j}\right]$ be an $m \times n$ real matrix with row vectors $r_{1}, r_{2}, \ldots, r_{m}$. We call $A$ is contractible on column (resp. row) $k$ if column (resp. row) $k$ contains exactly two nonzero entries. Suppose $A$ is contractible on column $k$ with $a_{i k} \neq$ $0 \neq a_{j k}$ and $i \neq j$. Then the $(m-1) \times(n-1)$ matrix $A_{i j: k}$ obtained from $A$ by replacing row $i$ with $a_{j k} r_{i}+a_{i k} r_{j}$, and deleting row $j$ and column $k$, is called the contraction of $A$ on column $k$ relative to rows $i$ and $j$. If $A$ is contractible on row $k$ with $a_{k i} \neq 0 \neq a_{k j}$ and $i \neq j$, then the matrix $A_{k: i j}=\left[A_{i j: k}^{T}\right]^{T}$ is called the contraction of $A$ on row $k$ relative to columns $i$ and $j$. We say that $A$ can be contracted to a matrix $B$ if either $B=A$ or there exist matrices $A_{0}, A_{1}, \ldots, A(t \geq 1)$ such that $A_{0}=A, A_{t}=B$, and $A_{r}$ is a contraction of $A_{r-1}$ for $r=1, \ldots, t$ (see [4]).

Let $A$ be an $n \times n$ tridiagonal matrix and $B$ be a contraction of $A$. Then in [5], authors proved that

$$
\operatorname{per} A=\operatorname{per} B
$$

There are many studies related with the permanents of the tridiagonal matrices connected with integer sequences. 
In [5], Kılıç et al. prove that the following $n \times n$ tridiagonal matrix holds

$$
\operatorname{per}\left(\begin{array}{ccccc}
1 & -1 & 0 & \cdots & 0 \\
-1 & 1 & -1 & \ddots & \vdots \\
0 & -1 & \ddots & \ddots & 0 \\
\vdots & \ddots & \ddots & \ddots & -1 \\
0 & \cdots & 0 & -1 & 1
\end{array}\right)=F_{n+1}
$$

where $F_{n}$ is the $n$th Fibonacci number. Moreover, Fibonacci sequence is named $A 000045$ in [1].

In [6], Lee et al. show that the permanent of $n \times n$ tridiagonal matrix in the form

$$
\left(\begin{array}{ccccc}
1 & 1 & 0 & \cdots & 0 \\
1 & 1 & 1 & \ddots & \vdots \\
0 & 1 & \ddots & \ddots & 0 \\
\vdots & \ddots & \ddots & \ddots & 1 \\
0 & \cdots & 0 & 1 & 1
\end{array}\right)
$$

is equal to $F_{n+1}$ for $n \geq 1$.

Another example is given by Ylmaz et al. in [7]. They show that the permanent of $n \times n$ tridiagonal matrix in the form

$$
\left(\begin{array}{cccccc}
1 & 2 & 0 & \cdots & \cdots & 0 \\
1 & -1 & 2 & \ddots & & \vdots \\
0 & 1 & 1 & \ddots & \ddots & \vdots \\
\vdots & \ddots & \ddots & \ddots & 2 & 0 \\
\vdots & & \ddots & 1 & 1 & 2 \\
0 & \cdots & \cdots & 0 & 1 & 3
\end{array}\right)
$$

is equal to $J_{n+1}$ for $n>2$.

In [8], Jína et al., by generalizing the results in [5] and [6], show that the permanent of $n \times n$ tridiagonal matrix of the form

$$
\left(\begin{array}{ccccc}
1 & \frac{1}{x} & 0 & \cdots & 0 \\
x & 1 & \frac{1}{x} & \ddots & \vdots \\
0 & x & \ddots & \ddots & 0 \\
\vdots & \ddots & \ddots & \ddots & \frac{1}{x} \\
0 & \cdots & 0 & x & 1
\end{array}\right)
$$


is equal to $F_{n+1}$ for $n \geq 1$, where $0 \neq x \in \mathbb{C}$.

One can find more applications related with the permanents of the $n$-square matrices connected with integer sequences [9-15].

In this paper, we define two $n \times n$ tridiagonal matrix families. Then we show that the permanents of these matrices are equal to the terms of Pell and Jacobsthal sequences. At the end of the paper, we give two Maple 13 procedures that give connections between these permanents and integer sequences.

\section{Main Results}

In this section, we consider two $n \times n$ tridiagonal matrix families. Then we study the relationships between the permanents of these families and Pell and Jacobsthal sequences.

Given two complex numbers $x$ and $y$ satisfying $x y=1$. Let $H_{n}=\left(h_{i, j}\right)$ be an $n \times n$ tridiagonal matrix whose entries are

$$
h_{i, j}= \begin{cases}2, & i=j, \\ x, & i=j+1 \\ y, & i=j-1 \\ 0, & \text { otherwise }\end{cases}
$$

That is,

$$
H_{n}=\left(\begin{array}{ccccc}
2 & y & 0 & \cdots & 0 \\
x & 2 & y & \ddots & \vdots \\
0 & x & \ddots & \ddots & 0 \\
\vdots & \ddots & \ddots & \ddots & y \\
0 & \cdots & 0 & x & 2
\end{array}\right)_{n \times n}
$$

Theorem 1. Let $x, y \in \mathbb{C}, x y=1$ and $H_{n}^{(r)}=\left(h_{i, j}^{(r)}\right)$ be the rth contraction of the matrix $H_{n}$ given by (2) for $1 \leq r \leq n-2$. Then

$$
h_{1, j}^{(r)}=\left\{\begin{array}{cl}
P_{r+2}, & j=1, \\
y P_{r+1}, & j=2, \\
0, & 3 \leq j \leq n-r
\end{array}\right.
$$

where $P_{n}$ is the nth Pell number. 
Proof. We prove the theorem by induction on $r$. Since the matrix $H_{n}$ can be contracted on column 1 so that

$$
\begin{aligned}
h_{1,1}^{(1)} & =h_{1,1} h_{2,2}+h_{2,1} h_{1,2} \\
& =2 \cdot 2+x y, \quad(x y=1) \\
& =5=P_{3} \\
h_{1,2}^{(1)} & =h_{1,1} h_{2,3}+h_{2,1} h_{1,3} \\
& =2 y+x \cdot 0 \\
& =y P_{2}
\end{aligned}
$$

and

$$
h_{1, j}^{(1)}=h_{1,1} h_{2, j+1}+h_{2,1} h_{1, j+1} .
$$

Observe that $h_{2, j+1}=h_{1, j+1}=0(j=3,4, \ldots, n-1)$. Consequently, we get $h_{1, j}^{(1)}=0$ for $3 \leq j \leq n-r$. The theorem holds for $r=1$.

Assume that the theorem holds for every $k, 2 \leq k \leq r$. Then we have to show that the theorem is true for $n+1$. Because of assumption we have

$$
H_{n}^{(r)}=\left(\begin{array}{ccccc}
P_{r+2} & y P_{r+1} & 0 & \cdots & 0 \\
x & 2 & y & \ddots & \vdots \\
0 & x & \ddots & \ddots & 0 \\
\vdots & \ddots & \ddots & \ddots & y \\
0 & \cdots & 0 & x & 2
\end{array}\right)_{(n-r) \times(n-r)}
$$

Since the matrix $H_{n}^{(r)}$ can be contracted on column 1 so that

$$
\begin{aligned}
h_{1,1}^{(r+1)} & =h_{1,1}^{(r)} h_{2,2}^{(r)}+h_{2,1}^{(r)} h_{1,2}^{(r)} \\
& =P_{r+2} \cdot 2+x\left(y P_{r+1}\right) \\
& =2 P_{r+2}=P_{r+1}(x y=1) \\
= & P_{r+3}, \\
h_{1,2}^{(r+1)} & =h_{1,1}^{(r)} h_{2,3}^{(r)}+h_{2,1}^{(r)} h_{1,3}^{(r)} \\
& =P_{r+2} \cdot y+x \cdot 0 \\
& =y P_{r+2},
\end{aligned}
$$


and

$$
h_{1, j}^{(r+1)}=h_{1,1}^{(r)} h_{2, j+1}^{(r)}+h_{2,1}^{(r)} h_{1, j+1}^{(r)} .
$$

Observe that $h_{2, j+1}^{(r)}=h_{1, j+1}^{(r)}=0(j=3,4, \ldots, n-r-1)$ from $(3)$.

Consequently, we obtain $h_{1, j}^{(r+1)}=0$ for $3 \leq j \leq n-r-1$. Thus the proof is completed.

Theorem 2. Let $x, y \in \mathbb{C}, x y=1$ and $H_{n}$ be the $n \times n$ tridiagonal matrix given by (2). Then

$$
\operatorname{per} H_{n}=P_{n+1}
$$

for $n \geq 1$, where $P_{n}$ is the nth Pell number.

Proof. Let $H_{n}^{(r)}$ be the $r$ th contraction of the matrix $H_{n}$. By Theorem 1 we have

$$
H_{n}^{(n-2)}=\left(\begin{array}{cc}
P_{n} & y P_{n-1} \\
x & 2
\end{array}\right)
$$

From (1) we obtain

$$
\begin{aligned}
\operatorname{per} H_{n} & =\operatorname{per} H_{n}^{(n-2)} \\
& =2 P_{n}+x y P_{n-1} \quad(x y=1) \\
& =P_{n+1},
\end{aligned}
$$

which is desired.

Given two complex numbers $z$ and $t$ satisfying $z t=2$. Let $K_{n}=\left(k_{i, j}\right)$ be an $n \times n$ tridiagonal matrix whose entries are

$$
k_{i, j}= \begin{cases}1, & i=j \\ z, & i=j+1 \\ t, & i=j-1 \\ 0, & \text { otherwise }\end{cases}
$$

That is,

$$
K_{n}=\left(\begin{array}{ccccc}
1 & t & 0 & \cdots & 0 \\
z & 1 & t & \ddots & \vdots \\
0 & z & \ddots & \ddots & 0 \\
\vdots & \ddots & \ddots & \ddots & t \\
0 & \cdots & 0 & z & 1
\end{array}\right)_{n \times n}
$$

By applying successive contractions according to first column of $K_{n}$, we reach the following theorem. 
Theorem 3. Let $z, t \in \mathbb{C}, z t=2$ and $K_{n}^{(r)}=\left(k_{i, j}^{(r)}\right)$ be the rth contraction of the matrix $K_{n}$ given by (4) for $1 \leq r \leq n-2$. Then

$$
k_{1, j}^{(r)}=\left\{\begin{array}{cl}
J_{r+2}, & j=1 \\
t J_{r+1}, & j=2, \\
0, & 3 \leq j \leq n-r
\end{array}\right.
$$

where $J_{n}$ is the nth Jacobsthal number.

With the help of Theorem 3 we present the following result that gives terms of the Jacobsthal sequence.

Theorem 4. Let $z, t \in \mathbb{C}, z t=2$ and $K_{n}$ be the $n \times n$ tridiagonal matrix given by (4). Then

$$
\operatorname{per} K_{n}=J_{n+1}
$$

for $n \geq 1$, where $J_{n}$ is the nth Jacobsthal number.

Proof. Let $K_{n}^{(r)}$ be the $r$ th contraction of the matrix $K_{n}$. By Theorem 3 we have

$$
K_{n}^{(n-2)}=\left(\begin{array}{cc}
J_{n} & t J_{n-1} \\
z & 1
\end{array}\right) .
$$

From (1) we obtain

$$
\begin{aligned}
\operatorname{per} K_{n} & =\operatorname{per} K_{n}^{(n-2)} \\
& =J_{n}+z t J_{n-1} \quad(z t=2) \\
& =J_{n+1},
\end{aligned}
$$

which is desired.

\section{Numerical Examples}

In this section, we present some examples related with the permanents of the matrices, generate the Pell and Jacobsthal numbers, obtained for specific values of $x, y, z$ and $t$ given in (2) and (4).

Example 5. Let $n \geq 1$ and $A_{n}$ be an $n \times n$ tridiagonal matrix where all terms on the main diagonal are 2 and all terms on the subdiagonal and 
superdiagonal are 4 and $\frac{1}{4}$ respectively; therefore,

$$
A_{n}=\left(\begin{array}{ccccc}
2 & \frac{1}{4} & 0 & \cdots & 0 \\
4 & 2 & \frac{1}{4} & \ddots & \vdots \\
0 & 4 & \ddots & \ddots & 0 \\
\vdots & \ddots & \ddots & \ddots & \frac{1}{4} \\
0 & \cdots & 0 & 4 & 2
\end{array}\right)
$$

Let $i=\sqrt{-1}$ and $B_{n}$ be an $n \times n$ tridiagonal matrix where all terms on the main diagonal are 2 and all terms on the subdiagonal and superdiagonal are $-7 i$ and $\frac{i}{7}$, respectively; therefore,

$$
B_{n}=\left(\begin{array}{ccccc}
2 & \frac{i}{7} & 0 & \cdots & 0 \\
-7 i & 2 & \frac{i}{7} & \ddots & \vdots \\
0 & -7 i & \ddots & \ddots & 0 \\
\vdots & \ddots & \ddots & \ddots & \frac{i}{7} \\
0 & \cdots & 0 & -7 i & 2
\end{array}\right)
$$

Investigating the first few permanents of the matrices $A_{n}$ and $B_{n}$ we get

$$
\begin{array}{ll}
\operatorname{per} A_{1}=2, & \operatorname{per} B_{1}=2, \\
\operatorname{per} A_{2}=\operatorname{per}\left(\begin{array}{cc}
2 & \frac{1}{4} \\
4 & 2
\end{array}\right)=5, \quad \operatorname{per} B_{2}=\operatorname{per}\left(\begin{array}{cc}
2 & \frac{i}{7} \\
-7 i & 2
\end{array}\right)=5, \\
\operatorname{per} A_{3}=\operatorname{per}\left(\begin{array}{lll}
2 & \frac{1}{4} & 0 \\
4 & 2 & \frac{1}{4} \\
0 & 4 & 2
\end{array}\right)=12, & \operatorname{per} B_{3}=\operatorname{per}\left(\begin{array}{ccc}
2 & \frac{i}{7} & 0 \\
-7 i & 2 & \frac{i}{7} \\
0 & -7 i & 2
\end{array}\right)=12 .
\end{array}
$$

The sequences of the permanents appear to be equal to the terms of the Pell sequence.

Example 6. Let $n \geq 1$ and $C_{n}$ be an $n \times n$ tridiagonal matrix where all terms on the main diagonal are 1 and all terms on the subdiagonal and 
superdiagonal are 2 and 1 , respectively; so

$$
C_{n}=\left(\begin{array}{ccccc}
1 & 1 & 0 & \cdots & 0 \\
2 & 1 & 1 & \ddots & \vdots \\
0 & 2 & \ddots & \ddots & 0 \\
\vdots & \ddots & \ddots & \ddots & 1 \\
0 & \cdots & 0 & 2 & 1
\end{array}\right)
$$

Let $i=\sqrt{-1}$ and $D_{n}$ be an $n \times n$ tridiagonal matrix where all terms on the main diagonal are 1 and all terms on the subdiagonal and superdiagonal are $-i$ and $2 i$, respectively; so

$$
D_{n}=\left(\begin{array}{ccccc}
1 & 2 i & 0 & \cdots & 0 \\
-i & 1 & 2 i & \ddots & \vdots \\
0 & -i & \ddots & \ddots & 0 \\
\vdots & \ddots & \ddots & \ddots & 2 i \\
0 & \cdots & 0 & -i & 1
\end{array}\right)
$$

Investigating the first few permanents of the matrices $C_{n}$ and $D_{n}$ we get

$$
\begin{array}{ll}
\operatorname{per} C_{1}=1, & \operatorname{per} D_{1}=1, \\
\operatorname{per} C_{2}=\operatorname{per}\left(\begin{array}{ll}
1 & 1 \\
2 & 1
\end{array}\right)=3, & \operatorname{per} D_{2}=\operatorname{per}\left(\begin{array}{cc}
1 & 2 i \\
-i & 1
\end{array}\right)=3, \\
\operatorname{per} C_{3}=\operatorname{per}\left(\begin{array}{lll}
1 & 1 & 0 \\
2 & 1 & 1 \\
0 & 2 & 1
\end{array}\right)=5, & \operatorname{per} D_{3}=\operatorname{per}\left(\begin{array}{ccc}
1 & 2 i & 0 \\
-i & 1 & 2 i \\
0 & -i & 1
\end{array}\right)=5 .
\end{array}
$$

The sequences of the permanents appear to be equal to the terms of the Jacobsthal sequence.

Example 7. Let $n \geq 1$ and $R_{n}$ be an $n \times n$ tridiagonal matrix where all terms on the main diagonal are 1 and all terms on the subdiagonal and superdiagonal are 3 and $\frac{2}{3}$ respectively; therefore,

$$
R_{n}=\left(\begin{array}{ccccc}
1 & \frac{2}{3} & 0 & \cdots & 0 \\
3 & 1 & \frac{2}{3} & \ddots & \vdots \\
0 & 3 & \ddots & \ddots & 0 \\
\vdots & \ddots & \ddots & \ddots & \frac{2}{3} \\
0 & \cdots & 0 & 3 & 1
\end{array}\right) .
$$


Let $i=\sqrt{-1}$ and $S_{n}$ be an $n \times n$ tridiagonal matrix where all terms on the main diagonal are 1 and all terms on the subdiagonal and superdiagonal are $-5 i$ and $\frac{2 i}{5}$, respectively; therefore,

$$
S_{n}=\left(\begin{array}{ccccc}
1 & \frac{2 i}{5} & 0 & \cdots & 0 \\
-5 i & 1 & \frac{2 i}{5} & \ddots & \vdots \\
0 & -5 i & \ddots & \ddots & 0 \\
\vdots & \ddots & \ddots & \ddots & \frac{2 i}{5} \\
0 & \cdots & 0 & -5 i & 1
\end{array}\right)
$$

The first few permanents of the matrices $R_{n}$ and $S_{n}$ are per $R_{1}=\operatorname{per} S_{1}=$ $1, \operatorname{per} R_{2}=\operatorname{per} S_{2}=3, \operatorname{per} R_{3}=\operatorname{per} S_{3}=5$ and $\operatorname{per} R_{4}=\operatorname{per} S_{4}=11$.

The permanents of these matrices generate the terms of the Jacobsthal sequence.

\section{Appendix A}

Following Maple 13 procedure gives the matrix $H_{n}$ given by (2), the $r$ th contraction of the matrix $H_{n}\left(H_{n}^{(r)}\left(H_{n}^{(0)}=H_{n}\right)\right)$ for $1 \leq r \leq n-2$ in Theorem 1 and the $(n+1)$ th term of Pell sequences.

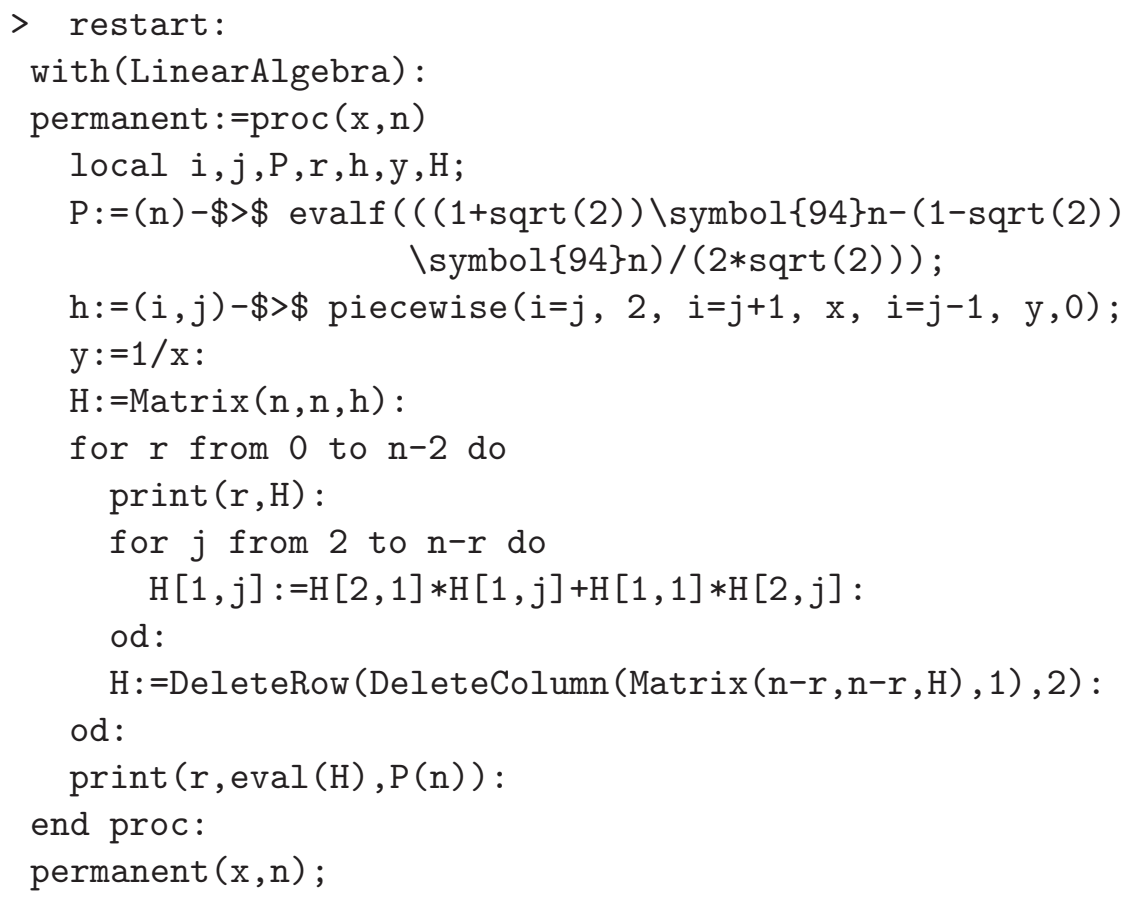




\section{Appendix B}

Following Maple 13 procedure gives the matrix $K_{n}$ given by (4), the $r$ th contraction of the matrix $K_{n}\left(K_{n}^{(r)}\left(K_{n}^{(0)}=K_{n}\right)\right)$ for $1 \leq r \leq n-2$ in Theorem 3 and the $(n+1)$ th term of Jacobsthal sequences.

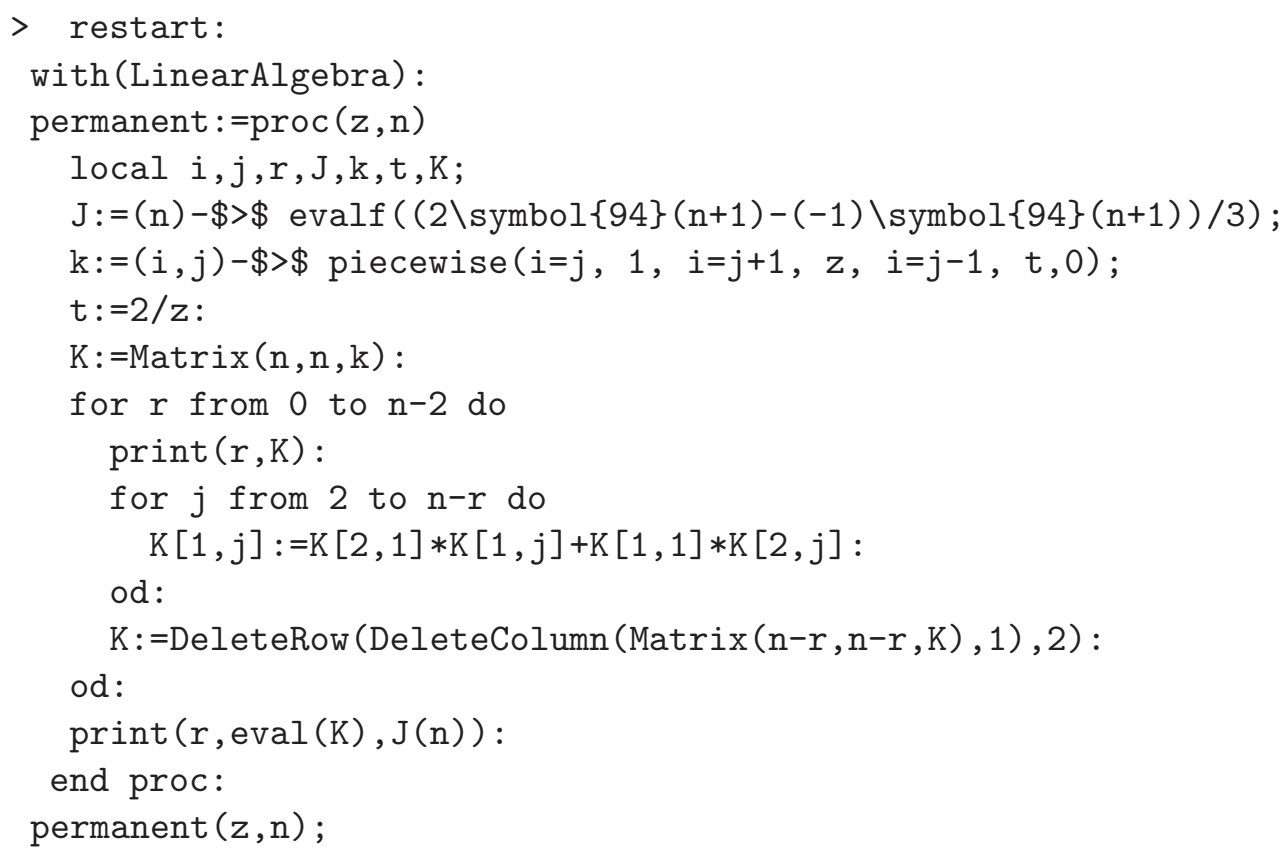

\section{References}

[1] The OEIS Foundation Inc., The On-Line Encyclopedia of Integer Sequences, http://oeis.org (2013).

[2] M. Marcus and H. Minc, Permanents, Amer. Math. Monthly, 72 (1965), 577-591.

[3] H. Minc, Encyclopedia of Mathematics and Its Applications, Permanents, AddisonWesley Publishing Company, London, (1978).

[4] R.A. Brualdi and P. M. Gibson, Convex polyhedra of doubly stochastic matrices I: Applications of the permanent function, J. Combin. Theory, Ser. A, 22 (1977), 194-230.

[5] E. Kılıç and D. Taşçi, On the permanents of some tridiagonal matrices with applications to the Fibonacci and Lucas numbers, Rocky Mountain J. Math., 37(6) (2007), 1953-1969.

[6] G.Y. Lee, S.G. Lee, H.G. Shin, On the $k$-generalized Fibonacci matrix $Q_{k}$, Linear Algebra Appl., 251 (1997), 73-88.

[7] F. Yılmaz, D. Bozkurt, The Adjacency Matrix of One Type of Directed Graph and the Jacobsthal Numbers and Their Determinantal Representation, J. Appl. Math., 2012 (2012), Article ID 423163. 
[8] J. Jína, P. Trojovský, On permanents of some tridiagonal matrices connected with Fibonacci Numbers, Int. J. Pure Appl. Math., 97(1) (2014), 79-87.

[9] M. Akbulak, A. Öteleş, On the number of 1-factors of bipartite graphs, Math. Sci. Lett., 2(3) (2013), 1-7.

[10] M. Akbulak, A. Öteleş, On the sum of Pell and Jacobsthal numbers by matrix method, Bull. Iranian Math. Soc., 40(4) (2014), 1017-1025.

[11] E. Kılıç and D. Taşçı, On the second order linear recurrences by tridiagonal matrices, Ars Combin., 91 (2009), 11-18.

[12] E. Kılıç, On the usual Fibonacci and generalized order-k Pell numbers, Ars Combin., 88 (2008), 33-45.

[13] K. Kaygisiz, A. Sahin, Determinant and Permanent of Hessenberg Matrix and Fibonacci Type Numbers, Gen. Math. Notes, 9(2) (2012), 32-41.

[14] K. Kaygisiz, A. Sahin, Determinants and permanents of Hessenberg matrices and generalized Lucas polynomials, Bull. Iranian Math. Soc., 39(6) (2013), 1065-1078.

[15] G.Y. Lee, k-Lucas numbers and associated bipartite graphs, Linear Algebra Appl., 320 (2000), 51-61. 